\title{
Inhomogeneous Extra Space as a Tool for the Top-Down Approach
}

\author{
Sergey G. Rubin $\mathbb{D}^{1,2}$ \\ ${ }^{1}$ National Research Nuclear University "MEPhI" (Moscow Engineering Physics Institute), Moscow, Russia \\ ${ }^{2}$ N.I. Lobachevsky Institute of Mathematics and Mechanics, Kazan Federal University, Kremlevskaya Street 18, Kazan 420008, Russia \\ Correspondence should be addressed to Sergey G. Rubin; sergeirubin@list.ru
}

Received 28 December 2017; Accepted 6 February 2018; Published 12 March 2018

Academic Editor: Elias C. Vagenas

Copyright (c) 2018 Sergey G. Rubin. This is an open access article distributed under the Creative Commons Attribution License, which permits unrestricted use, distribution, and reproduction in any medium, provided the original work is properly cited. The publication of this article was funded by $\mathrm{SCOAP}^{3}$.

\begin{abstract}
The top-down approach for the 6-dimensional space has been elaborated. The connection between the cosmological constant and the extra space metric has been obtained. The metric can be found with the necessary accuracy. It is shown that descent from high energies to the low ones leads to the quantum corrections which influence weakly the metric of extra space.
\end{abstract}

\section{Introduction}

Nowadays it becomes more or less clear that the physical laws are formed at high energies where we may only guess about the Lagrangian structure $[1,2]$. It is assumed that the values of observable parameters are the result of the evolution of our Universe started at high energies. Observed low-energy physics depend on parameters and initial conditions which have been formed at high energies $[3,4]$.

The natural values of the physical parameters are assumed to be quantities of the order of the Planck scale. At the same time, the observed parameter is determined at low energies, and their values are concentrated around electroweak scales and below. The ratio of these two scales is a small parameter, which creates difficulties in constructing the primary theory at high energy. Many attempts have been made to reconcile these two contradictory positions, but the skepticism of the scientific community remains. Quantum corrections only aggravate the situation.

In this article two ideas are attracted to soften the problem. Firstly, the connection between the primary physical parameters and the observable ones is achieved by suitable choice of an extra space metric. Secondly, we determine parameters of Lagrangian at a high-energy scale in the spirit of the effective field theory. In this case, quantum corrections being applied to primary parameters do not spoil the result.
One of the aims of the fundamental physics is to postulate a Lagrangian depending on primary parameters and find them using their connection with observational values. Suppose that one managed to obtain a set of relationships

$$
g_{k}=\Phi_{k}\left(\lambda_{i}(M)\right), \quad k=1,2, \ldots, K ; i=1,2, \ldots, I
$$

between primary parameters $\lambda_{i}(M)$ at an energy scale $M$ and the observational parameters $g_{k}$ (the particle masses, coupling constants, etc.) at low energies. Solving these equations with appropriate precision, one could determine primary parameters $\lambda_{i}(M)$ at a chosen scale $M$. The implementation of this plan in its entirety is a matter for the future. Nevertheless, an activity in this direction is observed. In the paper [5], warped geometry is used for the solution of the small cosmological constant problem. The hybrid inflation [6] has been developed to avoid the smallness of the inflaton mass. The electron-to-proton mass ratio is discussed in [7]. The seesaw mechanism is usually applied to explain the smallness of neutrino-to-electron mass ratio [8].

The aim of this paper is to establish and analyze only one connection

$$
\Lambda_{\mathrm{obs}}=\Phi\left(\lambda_{i}(M)\right)
$$

that ought to be considered as small but necessary part of a future theory. It has been proved earlier $[9,10]$ that there 
exists a set of primary parameters that are responsible for the observable value $\Lambda_{\text {obs }}$ of the cosmological constant (CC). Here much attention is paid to the problem of quantum corrections.

It is shown that the idea on an extra space existence facilitates connection of high-energy Lagrangian structure and the low-energy one. The observational smallness of the $\mathrm{CC}$ is used to find the extra space metric.

As a mathematical tool, we use the effective field theory technique, a well-known method for theoretical investigation of the energy dependence of physical parameters [11]. In this approach, parameters $\lambda_{i}(M)$ of the Wilson action are fixed at a high-energy scale $M$ and the renormalization flow is used to descend to low energies (the top-down approach) [12-15]. As is usually stated, the parameters $\lambda_{i}(M)$ of the Wilson action already contain quantum corrections caused by field fluctuations with energies between the chosen scale $M$ and maximal energy scale, the $D$-dimensional Planck mass $m_{D}$ in our case. Therefore the natural value of these parameters is $m_{D}$ that are usually many orders of magnitude greater than the electroweak scale $v \simeq 100 \mathrm{GeV}$.

The research is based on the multidimensional $f(R)$ gravity. The interest in $f(R)$ theories is motivated by inflationary scenarios starting with the work of Starobinsky [16]. The guiding principle underlying general relativity is the local invariance under coordinate transformations. We may use any invariant combination of quantities invariant under the general coordinate transformations keeping in mind two issues. Firstly, a theory must restore the Einstein-Hilbert action at low energies. Secondly, any gravitational action including the Einstein-Hilbert one is nonrenormalizable and should be considered as an effective theory.

The simplest extension of the gravitation theory is the one containing a function of the Ricci scalar $f(R)$. In the framework of such extension, many interesting results have been obtained. Some viable $f(R)$ models in 4-dim space that satisfies the observable constraints are proposed in [17-19]. Stabilization of extra space as the pure gravitational effect has been studied in $[20,21]$. It has been shown recently [9] that the $f(R)$ model with the deformed nonuniform extra space is able to reproduce the 4-dim Minkowski metric.

The extra dimensions have now become a widespread tool to obtain new theoretical results [22-25]. The idea of inhomogeneous extra space has been developed in [9, 26, 27] and plays one of the central roles in this research. It influences low-energy physics together with physical parameters of a Lagrangian. At the same time an accidental formation of manifolds with various metrics and topologies may be considered as a source of different universes whose variety is connected with a continuous set of extra space metrics. Entropic mechanism of a metric stabilization is considered in [28]. Stationary extra space metric is the final result of a metric evolution governed by the classical equation of motion, and hence the final stationary metric depends on initial configuration. One could keep in mind an analogy with the black hole mass where the Schwarzschild metric depends on an initial matter distribution. In the framework of the scalartensor theory, Weinberg [29] has proved that the firm finetuning of initial parameters of a Lagrangian is necessary if metric and scalar fields are constant in space-time. The latter means that the solution of the problem should be sought in the class of nonuniform configurations of metrics and fields. Metrics of the deformed extra space discussed in this paper belong to this class.

The plan of the paper consists of three steps. In Section 2, we consider a scalar field as the source of quantum corrections to the Lambda term. It will be shown that they are small relative to primary parameter value at high energies where physical parameters are fixed initially. The appropriate metric of inhomogeneous extra space is discussed in Section 3. In Section 4, the scalar field quantum corrections on the inhomogeneous background are analyzed.

\section{Quantum Corrections Caused by the Scalar Field: Minkowski Space}

The general goal of the top-down approach is to fix primary parameters by comparison with the experimental data at low energies. According to the effective field theory, quantum fluctuations with energies in the interval $\left(M, M_{P l}\right), M \ll$ $M_{P l}=1$, had been involved in the parameters of action. It means that their natural values are of the order of the Planck scale. Primary parameters are assumed to be formed at high energies, $M$ in our case.

Descending to the electroweak scale $v$ or lower where all physical parameters are measured is the necessary step. This process is accompanied by an alternation of physical parameters due to quantum fluctuations. The aim of this section is to demonstrate that quantum corrections are small. The toy model for the scalar field is considered to study the quantum corrections which are the result of integrating out the quick modes in the energy interval $(v, M), v \ll M$. The scalar field action is written in the standard form

$$
\begin{gathered}
S_{\chi}=\int d^{n} z\left[\frac{1}{2} \partial_{A} \chi g_{n}^{A B} \partial_{B} \chi-U(\chi ; \lambda)-c\right], \\
U(\chi ; \lambda)=\lambda_{2} \chi^{2}+\lambda_{4} \chi^{4}, \\
0<\lambda_{4}(M) \ll \lambda_{2}(M) \sim c(M) \sim 1
\end{gathered}
$$

acting in the ordinary 4-dimensional Minkowski space. In this section, we study quantum corrections to the parameter $c(M)$ which may be considered as the primary cosmological constant at the scale $M$.

The generating functional for action (3) at the scale $M$

$$
Z_{0}^{M}=\int_{0}^{M}[D \chi]_{M} \exp \left(i S_{\chi}\right)
$$

plays the central role in the effective field theory approach. Here and in the following a subscript and superscript indicate an interval of momentum in the Euclidean space $k_{E}$ that are taken into account. Thus, functional (5) is the result of integrating out quick modes $M<k_{E}<m_{D}$. The $D$-dimensional Planck mass $m_{D}$ is considered as the maximal energy scale in the rest of the article.

Let us integrate out modes with Euclidean momentum $k_{E}$ in the interval $v<k_{E}<M$ in generating functional 
(5) and shift down to the electroweak scale $v \sim 100 \mathrm{GeV}$. To this end, one should decompose the scalar field as follows:

$$
\chi(x)=\chi_{q}(x)+\chi_{s}(x)
$$

Here quick $\chi_{q}(x)$ and slow $\chi_{s}(x)$ modes in 4-dim Euclidean space are

$$
\begin{aligned}
& \chi_{q}(x)=\int_{k_{E}=v}^{k_{E}=M} \frac{d^{4} k_{E}}{(2 \pi)^{4}} e^{-i k_{E} x} \chi_{k_{E}}(x) ; \\
& \chi_{s}(x)=\int_{k_{E}=0}^{k_{E}=v} \frac{d^{4} k_{E}}{(2 \pi)^{4}} e^{-i k_{E} x} \chi_{k_{E}}(x)
\end{aligned}
$$

correspondingly.
Substituting (6) into (5) gives the generating functional in the form

$$
\begin{aligned}
Z_{0}^{M} & =Z_{0}^{v} \cdot \int_{v}^{M} D \chi_{q} \\
\cdot & \exp \left\{i \int d^{4} x \sqrt{g_{4}}\left[\frac{1}{2}\left(\partial \chi_{q}\right)^{2}-\lambda_{2} \chi_{q}^{2}-\delta U\left(\chi_{q}, \chi_{s}\right)\right]\right\}, \\
Z_{0}^{v} & =\int_{0}^{v} D \chi_{s} \exp \left\{i \int d^{4} x \sqrt{g_{4}}\left[\frac{1}{2}\left(\partial \chi_{s}\right)^{2}-U\left(\chi_{s} ; \lambda\right)\right]\right\},
\end{aligned}
$$

where

$$
\delta U\left(\chi_{q}, \chi_{s}\right)=4 \lambda_{4} \chi_{q}^{3} \chi_{s}+6 \lambda_{4} \chi_{q}^{2} \chi_{s}^{2}+4 \lambda_{4} \chi_{q} \chi_{s}^{3}+\lambda_{4} \chi_{q}^{4} .
$$

Here, we have taken into account orthogonality of $\chi_{s}$ and $\chi_{q}$. The way to integrate out the field $\chi_{q}$ from (8), provided that the coupling constant $\lambda_{4}$ is small, is well known (see, e.g., textbook [11]). Consider generating functional

$$
Z_{v}^{M}=\int_{v}^{M} D \chi_{q} \exp \left\{\int d^{4} x \sqrt{g_{4}}\left[\frac{1}{2}\left(\partial \chi_{q}\right)^{2}-\lambda_{2} \chi_{q}^{2}-\delta U\left(\chi_{q}, \chi_{s}\right)+\chi_{q}(x) J(x)\right]\right\}
$$

as a functional of an external current $J$. Then the result of integrating out quick modes is as follows:

$$
\begin{aligned}
Z_{v}^{M}= & e^{\epsilon-i \int d^{4} x\left[\delta U\left(-i \delta / \delta J(x), \chi_{s}\right)\right]} \\
& \cdot e^{(-i / 2) \int d^{4} x d^{4} x^{\prime} J(x) \Delta\left(x-x^{\prime}\right) J\left(x^{\prime}\right)}, \\
\epsilon \equiv & -\frac{1}{2} S p \ln \left(\square_{4}+2 \lambda_{2}\right)_{v}^{M}, \\
\Delta(x)= & \int \frac{d^{4} k}{(2 \pi)^{4}} \frac{e^{-i k x}}{k^{2}-2 \lambda_{2}+i \epsilon} .
\end{aligned}
$$

After the Wick rotation, quantum correction $\epsilon$ acquires the form

$$
\epsilon=-i \frac{V T}{16 \pi^{2}} \int_{v}^{M} d k_{E} k_{E}^{3} \ln \left(k_{E}^{2}+2 \lambda_{2}\right)
$$

and can be easily calculated. As a result, the contribution (14) to the bare cosmological constant $c(M)$

$$
\delta c=\frac{\epsilon}{i V T}=-\frac{M^{4}}{64 \pi^{2}} \ln \left(2 \lambda_{2}\right)+o\left(M^{4}\right)
$$

is small because of the inequality $M \ll 1$.

The integral in (14) is usually estimated keeping in mind that a cutoff scale is much greater than the Lagrangian parameters; that is, the inequality $M^{2} \gg \lambda_{2}$ holds; see recent discussion in [30]. First estimation has been presented by Zeldovich in 1967 [31], where the proton mass was used as the maximum energy scale. In our case, the situation is different. Indeed, the scale $M$ is chosen such that $M \ll 1$ while a natural value of the effective parameter $\lambda_{2} \sim 1$; see (4). In both cases, the corrections are proportional to the fourth power of the energy scale $M$. This is not surprising, since the chosen scale $M$ is still much larger than the electroweak scale $v$.

Estimation (15) for the quantum corrections $\delta c$ at the scale $M \sim 10^{15} \mathrm{GeV}$ gives

$$
\delta c \sim 10^{-19} \sim 10^{57} \mathrm{GeV}^{4} .
$$

This value is negligibly small as compared to the primary (bare) value $c \sim 1=\left(10^{19}\right)^{4} \mathrm{GeV}^{4}$ of the $\Lambda$ term

$$
\frac{\delta c}{c(M)} \ll 1
$$

and is huge as compared to the observational value. The latter is not a great problem if our intention is to find values of the physical parameters at a high-energy scale. Indeed, quantum corrections must be compared to primary, physical parameters rather than the observational ones.

It is interesting to check for future studies that correction to the mass $m=\sqrt{2 \lambda_{2}}$ also contains the small parameter $M$ and hence is small. To verify this, let us estimate quantum corrections produced by terms proportional to $\chi_{q}^{2}$. The latter can be extracted from (9) and (10) and has the form

$$
\delta U_{2} \equiv 6 \lambda_{4} \chi_{q}(x)^{2} \chi_{s}(x)^{2} .
$$

Receipt (11) with $\delta U_{2}$ instead of $\delta U$ leads to the quantum correction

$$
\delta U_{s}\left(\chi_{s}\right)=\int d^{4} x 6 \lambda_{4} \Delta(0) \chi_{s}(x)^{2}
$$

to the potential in the first multiplier $Z_{0}^{v}$ in expression (8). Here

$$
\Delta(0)=-\int \frac{d^{4} k_{E}}{(2 \pi)^{4}} \frac{1}{k_{E}^{2}+2 \lambda_{2}} \simeq-\frac{1}{64 \pi^{2}} \frac{M^{4}}{\lambda_{2}}
$$


for $M^{2} \ll \lambda_{2}$. This means that the quantum correction to $\lambda_{2}$

$$
\delta \lambda_{2}=6 \lambda_{4} \Delta(0)=-\frac{3 M^{4}}{32 \pi^{2}} \frac{\lambda_{4}}{\lambda_{2}}
$$

is small due to the last inequality in (4) and the choice of energy scale $M \ll 1$. Hence, the quantum correction $\delta m \sim$ $\delta \lambda_{2}$ to the mass $m$ is also small.

We can conclude that the quantum corrections are small in comparison with the primary physical parameter. The mass of the scalar particles remains on the order of the Planck scale, which only means that they cannot be created at low energies. A much more serious defect lies in the fact that it is not possible to neutralize the difference between the primary and observational values of CC. We must complicate the model to solve this problem.

To this end, one may draw on the method developed in articles $[9,10]$. As has been shown in [9], the problem can be strongly facilitated on the classical level by the 6-dim scalartensor gravity with higher derivatives. Moreover, the way of explanation of the CC smallness in the framework of pure gravity without scalar fields was studied in [10]. The latter is shortly discussed in the next section and the Appendix for clarity.

\section{Inclusion of Inhomogeneous Extra Space}

In this section, we shortly consider the connection of the CC value and the form of extra space. The discussion is performed on the classical level while the quantum corrections are considered in the next section. Following the ideas developed in $[9,10]$, consider $f(R)$ gravity with the action

$$
\begin{aligned}
S_{g} & =\frac{m_{D}^{4}}{2} \int d^{6} z \sqrt{-g_{6}} f(R), \\
f(R) & =R+a R^{2}+c
\end{aligned}
$$

acting in a 6-dim space. The constant $c$ that was written explicitly in (3) is involved now in the definition of the function $f(R)$.

The metric is assumed to be the direct product $M_{4} \times V_{2}$ of the 4-dim space $M_{4}$ and 2-dim compact space $V_{2}$

$$
\begin{aligned}
d s^{2} & =g_{6, A B} d z^{A} d z^{B} \\
& =g_{4, \mu \nu}(x) d x^{\mu} d x^{\mu}+g_{2, a b}(y) d y^{a} d y^{b} .
\end{aligned}
$$

Here, $g_{4, \mu \nu}(x)$ and $g_{2, a b}(y)$ are metrics of the manifolds $M_{4}$ and $V_{2}$, respectively. $x$ and $y$ are the coordinates of the subspaces $M_{4}$ and $V_{2}$. We will refer to 4 -dim space $M_{4}$ and 2dim compact space $V_{2}$ as the main space and an extra space, respectively. The metric has the signature $(+---\cdots)$, and the Greek indexes $\mu, v=0,1,2,3$ refer to 4 -dimensional coordinates. Latin indexes run over $a, b=4,5$.

There are three scales of energy: the 6-dim Planck mass $m_{D}$, the characteristic size $r_{c}$ of the compact extra space $V_{2}$, and the low-energy scale $v \sim 100 \mathrm{GeV}$. It is assumed that the scales $m_{D}, r_{c}^{-1}$, and $v$ satisfy the inequalities

$$
v \ll r_{c}^{-1} \ll m_{D}=1 .
$$

The first inequality in (25) means that a characteristic energy scale of extra space is large (the experimental limit is $r_{c}^{-1} \geq 10^{4} \mathrm{GeV}$ ) and its geometry is stabilized shortly after the Universe creation [21, 22, 28, 32-34]. On the other side, quantum behavior dominates at the $m_{D}$ scale and if one intends to describe a metric of extra space classically, the second inequality must take place. In the following, everything is measured in the $m_{D}$ units.

As will be shown later, condition

$$
v \ll M \ll r_{c}^{-1}
$$

for the energy scale $M$ is an appropriate choice. Indeed, the inequality $v \ll M$ permits us to consider the masses of particles to be zero. At the same time, excitations of compact extra space geometry are known to form the Kaluza-Klein tower with energies $E>r_{c}^{-1}$. If we start from the energy scale $M \ll r_{c}^{-1}$, the excitations are suppressed and extra space metric $g_{2, a b}$ represents a stationary configuration described by (ab) part of the classical equations of motion

$$
\begin{gathered}
R_{A B} f^{\prime}-\frac{1}{2} f(R) g_{6, A B}+\nabla_{A} \nabla_{B} f_{R}-g_{6, A B} \square f^{\prime}=0, \\
+ \text { additional conditions, }
\end{gathered}
$$

where $\square f_{R}=\nabla^{A} \nabla_{A} f_{R}$.

Let us assume that the metric of our 4-dim space is the Minkowski metric, $g_{4}=\operatorname{diag}(1,-1,-1,-1)$. The compact 2 dim manifold is supposed to be parameterized by the two spherical angles $y_{1}=\theta$ and $y_{2}=\phi(0 \leq \theta \leq \pi, 0 \leq \phi<2 \pi)$. The choice of the extra space metric is as follows:

$$
\begin{aligned}
& g_{2, \theta \theta}=-r(\theta)^{2} ; \\
& g_{2, \phi \phi}=-r(\theta)^{2} \sin ^{2}(\theta) .
\end{aligned}
$$

There is continuous set of extra space metrics, solutions to the differential equations (27), characterized by additional conditions. Maximally symmetrical extra spaces that are used in great majority of literature represent a small subset of this set. As additional conditions, we fix the metric at the point $\theta=\pi$

$$
\begin{aligned}
& r(\pi)=r_{\pi} \\
& r^{\prime}(\pi)=0 \\
& R(\pi)=R_{\pi} \\
& R^{\prime}(\pi)=0
\end{aligned}
$$

The system of equations (27) together with these conditions completely determines the form of extra space metric.

Numerical solutions to (27) with additional conditions (29) are discussed in $[9,26]$. It has been found that, due to high nonlinearity of the equation, the gravity can trap itself in a small region around $\theta=0$ even without matter contribution.

The next step consists of finding an appropriate 2-dim extra metric with the help of the observable value of CC. 


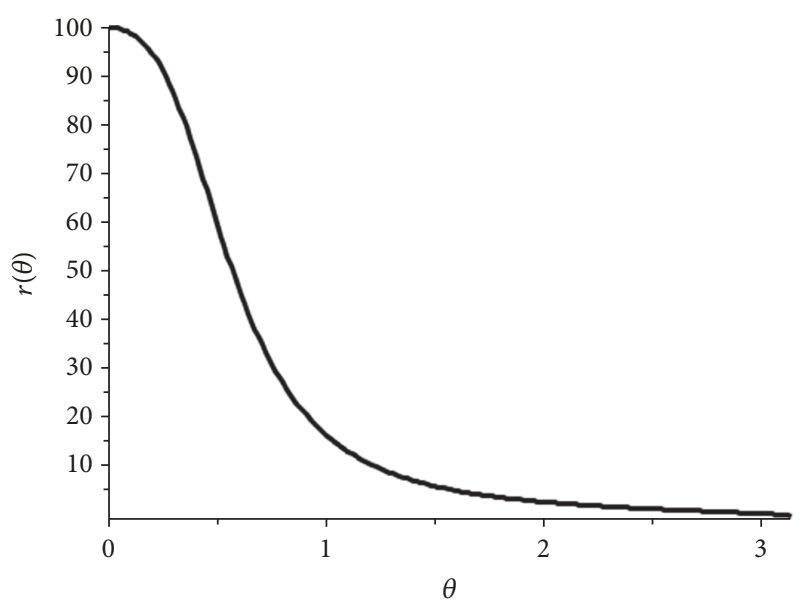

(a)

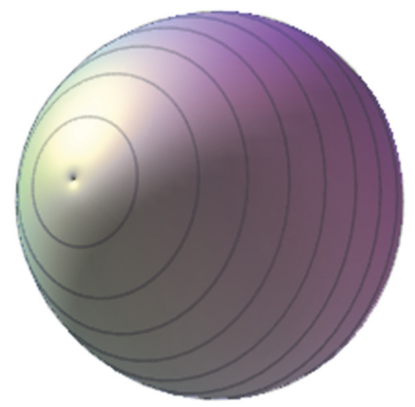

(b)

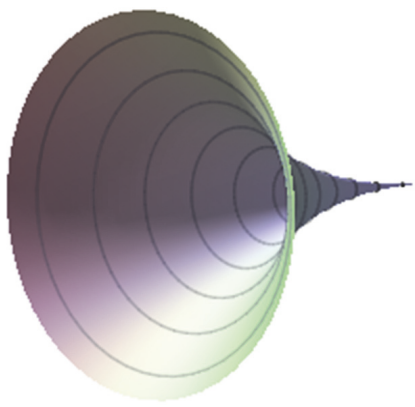

(c)

Figure 1: (a) Radius $r(\theta)$ of extra space for the parameter values $a=-100, c=-2.1 \cdot 10^{-3}$ and additional conditions $r(\pi)=100, R(\pi)=$ $1.0251 \cdot 10^{-3}$. (b) 3D plot of the solution. (c) A small part of the 3D plot near $\theta=0$ (left "end").

General connection is represented in the Appendix, formula (A.4). It should be stressed that our aim is not to calculate CC with extremal accuracy $10^{-123}$ but to find physical parameters at high-energy scale $M$. In this case, the left hand side of (A.4) can be safely substituted by zero and we arrive at the following connection between the physical parameters:

$$
\begin{aligned}
& \Lambda_{\text {theor }}(a, c, r(\pi), R(\pi)) \\
& \equiv-\frac{\pi}{M_{P l}^{2}} \int d \theta \sqrt{\left|g_{2}(\theta)\right|} f\left(R_{2}(\theta)\right) \simeq 0 .
\end{aligned}
$$

To be more specific, suppose that the primary parameters of the Lagrangian and the extra space size dictated by the parameter $r(\pi)$ are known:

$$
\begin{aligned}
a & =-100, \\
c & =-2.1 \cdot 10^{-3}, \\
r(\pi) & =100 .
\end{aligned}
$$

Then, numerical solution to (30) with respect to the Ricci scalar $R(\pi)$ can be obtained:

$$
R(\pi) \simeq 1.0251 \cdot 10^{-3} .
$$

Relative accuracy $10^{-4}$ of this result can be improved if necessary. Thus, we have found the extra space metric (see Figure 1) of our toy model with appropriate precision.

The 4-dim Planck mass can be found numerically according to expression (A.5) and equals $M_{P l}=34 m_{D}$. This gives the value of the $D$-dim Planck mass, $m_{D} \simeq 3 \cdot 10^{17} \mathrm{GeV}$. Therefore the scale $M$ may be chosen in the interval $10^{2} \ll$ $M \ll 10^{17} \mathrm{GeV}$; see inequality (26).

The intermediate conclusion is that the smallness of CC can be used at the classical level for fixing appropriate metric of the inhomogeneous extra space. In the next section, we discuss the role of quantum corrections and their influence on this classical result.

\section{Quantum Corrections Caused by the Scalar Field: Inhomogeneous Extra Space Background}

In this section, we discuss the way to integrate out the extra space coordinates in expression (3) to obtain an effective 4dim action describing physics at the scale $M$. Information about the extra space metric will be stored in the effective parameters at this scale.

As discussed below formula (26), the excitations of the extra space metric are suppressed due to the choice of the scale $M$. The same arguments may be applied to the scalar field excitations on the 2-dim extra space. Classical distribution can be obtained from the equation of motion

$$
\square_{6} \varphi+U^{\prime}(\varphi ; \lambda)=0,
$$

where $\square_{6}$ is 6-dim d'Alemert operator. Let us decompose the scalar field into a series of orthonormal functions $Y_{n}$ acting on the extra space. The smallness of fluctuations at the scale $M$ means that we may limit ourselves to the first term

$$
\varphi(x, \theta)=\chi(x) Y(\theta),
$$

where $Y(\theta)$ is a solution to classical equation

$$
\square_{2} Y(\theta)+2 \lambda_{2} Y(\theta)=0 .
$$

This equation is obtained from (33) by neglecting small terms containing $x$-derivatives (see Appendix, formula (A.1)) and those terms proportional to the small coupling $\lambda_{4}$. More explicit form of this equation

$$
\cot (\theta) \partial_{\theta} Y(\theta)+\partial_{\theta}^{2} Y(\theta)-2 \lambda_{2} r(\theta)^{2} Y(\theta)=0
$$

can be obtained by substituting metric (28) into (35). Approximate solution to this equation

$$
Y(\theta)=C e^{-\sqrt{2 \lambda_{2}} \int_{0}^{\theta} d \theta^{\prime} r\left(\theta^{\prime}\right)}
$$


has been found in [9]. Here,

$$
C=\left[2 \pi \int d \theta r(\theta)^{2} \sin \theta e^{-2 \sqrt{2 \lambda_{2}} \int_{0}^{\theta} d \theta^{\prime} r\left(\theta^{\prime}\right)}\right]^{-1 / 2}
$$

is the normalization constant.

After substituting (34) into expression (3), we get the following form of the effective 4-dim action for the gravity with the scalar field

$$
\begin{aligned}
S & =S_{g}+S_{\chi}, \\
S_{\chi} & =\frac{1}{2} \int d^{4} x\left[\frac{1}{2} \partial_{\mu} \chi(x) g^{\mu \nu} \partial_{\nu} \chi(x)-U\left(\chi ; \lambda_{\mathrm{eff}}\right)\right],
\end{aligned}
$$

where

$$
\begin{aligned}
\lambda_{\mathrm{eff}, 2} & =\lambda_{2}-\frac{1}{2} \int d \theta \sqrt{\left|g_{2}(\theta)\right|} \partial_{a} Y(\theta) g_{2}^{a b}(\theta) \partial_{b} Y(\theta) \\
& \simeq 3 \lambda_{2}, \\
\lambda_{\mathrm{eff}, 4} & =\lambda_{4} \cdot \int d \theta \sqrt{\left|g_{2}(\theta)\right|} Y(\theta)^{4}
\end{aligned}
$$

and $S_{g}$ is the gravitational action (22). As was shown in Section 3, the extra space metric may be chosen such that the metric of our 4-dim space is arbitrarily close to the Minkowskian metric. The second equality in (40) is true due to the form of solution (37) and metric $g_{2}^{\theta \theta}(\theta)=$ $-r(\theta)^{-2}$. New effective parameters $\lambda_{\text {eff, } 2,4}$ depend on the functions $Y(\theta), g_{2, a b}(\theta)$ and, hence, on the additional conditions $r(\pi), R(\pi)$. A connection of effective 4 -dim parameters with the metric of extra space is the well-known result. The most known example is connection of the Planck mass to a $D$-dim Planck mass [32] $M_{P l}^{2}=m_{D}^{D-2} V_{e}$, where $V_{e}$ stands for an extra space volume.

The mass of the field $m_{\text {eff }}=\sqrt{2 \lambda_{\text {eff, } 2}}$ remains of the same order of the magnitude; $m_{\text {eff }} \sim m=\sqrt{2 \lambda_{2}}$. Specific example considered in Appendix gives $m \simeq 3 \cdot 10^{17} \mathrm{GeV}$. To perform numerical calculation of the coupling constant $\lambda_{4}$, let us use the metric presented in Figure 1. In this case, the integral in (41) can be evaluated and we obtain the renormalized parameter

$$
\lambda_{\mathrm{eff}, 4} \simeq 0.19 \lambda_{4}
$$

Therefore, the effective parameters satisfy the same inequalities as those mentioned in (4); $\lambda_{\text {eff }, 4} \ll \lambda_{\text {eff }, 2} \sim 1$.

We have restored the scalar field action (3) with the effective parameter values (40) and (41) depending on the extra space metric and the values of primary parameters. Therefore, the result of Section 2 may be applied to the action (39) to study the influence of quantum corrections.

The inclusion of the quantum corrections means that the bare value of the parameter $c$ should be substituted by $c+\delta c$ in (30):

$$
\Lambda_{\text {theor }}(a, c+\delta c, r(\pi), R(\pi))=0 .
$$

As was discussed above (see (17)), the ratio $\delta c / c$ is small. Therefore the shift $c \rightarrow c+\delta c$ can be easily compensated by a small shift in the Ricci scalar $R(\pi) \rightarrow R(\pi)+\delta R(\pi)$ in (43). This means that the quantum corrections lead only to a more accurate determination of the extra space metric depending on $R(\pi)$ in our case.

\section{Conclusion}

In this paper, the top-down approach for the 6-dim space has been elaborated. On the basis of toy model, the connection between the observable 4-dim Lambda term and the extra space metric $g_{2}$ has been obtained. It permits us to find this metric with an appropriate precision provided that other parameters are known.

Values of the physical parameters $\lambda_{i}(M)$ were chosen of the order of unity in 6-dim Planck units $m_{D}$ at the highenergy scale. It is shown here that the descent from the high-energy scale $M$ to the electroweak scale $v$ leads to the quantum corrections $\delta \lambda_{i}(M)$ which are small as compared to the primary (bare) values $\lambda_{i}(M)$. For specific case discussed in the article, the quantum corrections to the primary $\Lambda$ term permit us to calculate metric of the extra space more accurately.

The results of this research are valid in the energy interval $v \ll M \ll m_{D}$. If $M \sim m_{D}$, the quantum corrections are compatible with the classical results so that their influence cannot be controlled. If $M \lesssim v$, knowledge of the observable particle masses is necessary to evaluate integrals like those presented in (11) and (14).

The model discussed above contains the set of primary parameters $a, c, r(\pi), R(\pi)$. Connections for other low-energy physical parameters similar to (A.4) should be included if one wishes to determine all the primary parameters. This is the subject of future research. The discovery of the gravitational waves (GW) [35] provides an additional tool for such activity.

GW propagate from distant galaxies to the Earth with practically no distortion caused by interaction with the matter. In this regard, GW become a significant tool in the analysis of extra dimension properties. In the near future, some restrictions on the theory of gravity can be put when the number of GW sources amounts to several hundreds. Though many interesting results for sure will be obtained, one can foresee serious difficulties caused by a large number of models. The problem is that results depend on a structure of extra space, a number of extra dimensions, and their size. For example, interesting result has been obtained in [36] where the difference between propagations of GW and electromagnetic waves has been studied. This study is applicable only for a one-dimensional extra space.

The situation is aggravated by the fact that a lot of gravity theories other than the Einstein-Hilbert theory have been developed up to now. Specific choice of theory could produce additional effects like GW propagation with a speed different from the speed of light [37]. Nevertheless, new methods will undoubtedly help to extract promising directions in gravitational physics. For example, the methodologies that depend on redshift [38] or the ones that are valid at short distances for $z \ll 1$ [39] have been elaborated. Also, substantial review can be found in [40]. 


\section{Appendix}

Here the explicit form of relationship (30) between the Lambda term and the primary parameters (23) is found. Throughout the paper, the metric of our 4-dim space is the (almost) Minkowski one, $g_{4} \cong \operatorname{diag}(1,-1,-1,-1)$. It means that the Ricci scalar $R_{4}$ of our space is small compared to the Ricci scalar $R_{2}$ of the extra space. More definitely, the inequalities

$$
\begin{aligned}
& R_{4} \ll R_{2}, \\
& \partial_{\mu} \ll \partial_{a}, \quad \mu=0,1,2,3, a=4,5
\end{aligned}
$$

take place if one takes into account the experimental limit on the extra space size $L_{n}<10^{-18} \mathrm{~cm}$ and connection $R_{2} \sim 1 / L_{2}^{2}$ between the size $L_{2}$ and the Ricci scalar of the extra space. More discussion may be found in [21, 41].

Let us transform the gravitational part of the action. Using inequality (A.1), the Taylor expansion $f(R)=f\left(R_{4}+R_{2}\right) \simeq$ $f\left(R_{2}\right)+f^{\prime}\left(R_{2}\right) R_{4}$ in expression (3) gives [21]

$$
\begin{aligned}
S_{g} & =\pi \int d^{4} x d \theta \sqrt{\left|g_{4}(x) g_{2}(\theta)\right|}\left[R_{4} f^{\prime}\left(R_{2}(\theta)\right)\right. \\
& \left.+f\left(R_{2}(\theta)\right)\right] .
\end{aligned}
$$

Comparison of expression (A.2) with the Einstein-Hilbert action

$$
S_{\mathrm{EH}}=\frac{M_{P l}^{2}}{2} \int d^{4} x \sqrt{\left|g_{4}(x)\right|}\left(R_{4}-2 \Lambda_{\mathrm{obs}}\right)
$$

leads to the relationship

$$
\begin{aligned}
\Lambda_{\text {obs }} & =\Lambda_{\text {theor }}(a, c, r(\pi), R(\pi)) \\
& \equiv-\frac{\pi}{M_{P l}^{2}} \int d \theta \sqrt{\left|g_{2}(\theta)\right|} f\left(R_{2}(\theta)\right)
\end{aligned}
$$

that connects the observable value $\Lambda_{\text {obs }}$ of the CC with four main parameters of the model, $a, c$ from expression (23) and the parameters $r(\pi), R(\pi)$ characterizing the extra space metric (29). Here, $M_{P l}$ is the 4-dim Planck mass. The relationship (A.4) represents the particular case of connection (30). Its r.h.s. depends also on a stationary geometry $g_{2, a b}(\theta)$ and hence on the flexible parameters $R(\pi)$ and $r(\pi)$. Comparison of expression (A.2) with the Einstein-Hilbert action (A.3) gives also the Planck mass

$$
M_{P l}^{2}=2 \pi \int d \theta \sqrt{\left|g_{2}(\theta)\right|} f^{\prime}\left(R_{2}(\theta)\right)
$$

as the function of the primary parameters and geometry of the extra space.

\section{Conflicts of Interest}

The author declares that there are no conflicts of interest regarding the publication of this paper.

\section{Acknowledgments}

The author is grateful to I. Buchbinder and D. Kazakov for interesting discussions and O. Moiseeva for valuable remarks. This work was supported by the MEPhI Academic Excellence Project (agreement with the Ministry of Education and Science of the Russian Federation, August 27, 2013, Project no. 02.a03.21.0005) and by the Russian Government Program of Competitive Growth of Kazan Federal University. The work was supported by the Ministry of Education and Science of the Russian Federation, Project no. 3.472.2017/K.

\section{References}

[1] R. H. Brandenberger, A. Nayeri, S. P. Patil, and C. Vafa, "String gas cosmology and structure formation," International Journal of Modern Physics A, vol. 22, no. 21, pp. 3621-3642, 2007.

[2] M. Tegmark, A. Aguirre, M. J. Rees, and F. Wilczek, "Dimensionless constants, cosmology, and other dark matters," Physical Review D: Particles, Fields, Gravitation and Cosmology, vol. 73, no. 2, Article ID 023505, 2006.

[3] A. Loeb, "An observational test for the anthropic origin of the cosmological constant," Journal of Cosmology and Astroparticle Physics, no. 5, article no. 009, 2006.

[4] A. Ashoorioon, K. Dimopoulos, M. M. Sheikh-Jabbari, and G. Shiu, "Reconciliation of high energy scale models of inflation with Planck," Journal of Cosmology and Astroparticle Physics, vol. 2014, no. 2, article no. 025, 2014.

[5] A. Krause, "A small cosmological constant and backreaction of non-finetuned parameters," Journal of High Energy Physics, vol. 09, article 016, 2003.

[6] A. Linde, "Hybrid inflation," Physical Review D: Particles, Fields, Gravitation and Cosmology, vol. 49, no. 2, pp. 748-754, 1994.

[7] O. L. Trinhammer, "On the electron to proton mass ratio and the proton structure," EPL (Europhysics Letters), vol. 102, no. 4, Article ID 42002, 2013.

[8] A. Ibarra, E. Molinaro, and S. T. Petcov, "Low energy signatures of the TeV scale seesaw mechanism," Physical Review D: Particles, Fields, Gravitation and Cosmology, vol. 84, Article ID 013005, 2011.

[9] S. G. Rubin, "Scalar field localization on deformed extra space," The European Physical Journal C, vol. 75, no. 7, 2015.

[10] K. Bronnikov, R. Budaev, A. Grobov, A. Dmitriev, and S. G. Rubin, "Inhomogeneous compact extra dimensions," Journal of Cosmology and Astroparticle Physics, vol. 2017, no. 10, pp. 001001, 2017.

[11] M. E. Peskin and D. V. Schroeder, An Introduction to Quantum Field Theory, Addison-Wesley, Reading, Mass, USA, 1995.

[12] C. P. Burgess, "The Cosmological Constant Problem: Why its hard to get Dark Energy from Micro-physics," in Proceedings of the 100th Les Houches Summer School: Post-Planck Cosmology, pp. 149-197, Les Houches, France, July 2013.

[13] M. P. Hertzberg and A. Masoumi, "Can compactifications solve the cosmological constant problem?" Journal of Cosmology and Astroparticle Physics, vol. 2016, no. 6, article no. 053, 2016.

[14] A. Babić, B. Guberina, R. Horvat, and H. Štefančić, "Renormalization-group running of the cosmological constant and its implication for the Higgs boson mass in the standard model," Physical Review D: Particles, Fields, Gravitation and Cosmology, vol. 65 , no. 8, 2002. 
[15] E. Dudas, C. Papineau, and V. Rubakov, "Flowing to four dimensions," Journal of High Energy Physics, no. 3, pp. 50575073, 2006.

[16] A. A. Starobinsky, "A new type of isotropic cosmological models without singularity," Physics Letters B, vol. 91, no. 1, pp. 99-102, 1980.

[17] A. De Felice and S. J. Tsujikawa, " $\mathrm{f}(\mathrm{R})$ theories," Living Reviews in Relativity, vol. 13, p. 3, 2010.

[18] K. Bamba, A. N. Makarenko, A. N. Myagky, S. Nojiri, and S. D. Odintsov, "Bounce cosmology from $\mathrm{F}(\mathrm{R})$ gravity and $\mathrm{F}(\mathrm{R})$ bigravity," Journal of Cosmology and Astroparticle Physics, vol. 2014, no. 1, article no. 008, 2014.

[19] L. M. Sokołowski, "Metric gravity theories and cosmology. II. Stability of a ground state in $f(R)$ theories," Classical and Quantum Gravity, vol. 24, no. 14, pp. 3713-3734, 2007.

[20] U. Günther, A. Zhuk, V. B. Bezerra, and C. Romero, "AdS and stabilized extra dimensions in multi-dimensional gravitational models with nonlinear scalar curvature terms $R^{-1}$ and $R^{4}$," Classical and Quantum Gravity, vol. 22, no. 16, pp. 3135-3167, 2005.

[21] K. A. Bronnikov and S. G. Rubin, "Self-stabilization of extra dimensions," Physical Review D: Particles, Fields, Gravitation and Cosmology, vol. 73, no. 12, Article ID 124019, 2006.

[22] R. B. Abbott, S. M. Barr, and S. D. Ellis, "Kaluza-Klein cosmologies and inflation," Physical Review D: Particles, Fields, Gravitation and Cosmology, vol. 30, no. 4, pp. 720-727, 1984.

[23] M. Chaichian and A. B. Kobakhidze, "Mass hierarchy and localization of gravity in extra time," Physics Letters B, vol. 488, no. 2, pp. 117-122, 2000.

[24] L. Randall and R. Sundrum, "An alternative to compactification," Physical Review Letters, vol. 83, no. 23, pp. 4690-4693, 1999.

[25] A. R. Brown, A. Dahlen, and A. Masoumi, "Compactifying de Sitter space naturally selects a small cosmological constant," Physical Review D: Particles, Fields, Gravitation and Cosmology, vol. 90, no. 12, Article ID 124048, 2014.

[26] V. A. Gani, A. E. Dmitriev, and S. G. Rubin, "Deformed compact extra space as dark matter candidate," International Journal of Modern Physics D, vol. 24, no. 13, Article ID 1545001, 2015.

[27] S. G. Rubin, "The role of initial conditions in the universe formation," Gravitation \& Cosmology, vol. 21, no. 2, pp. 143-151, 2015.

[28] A. A. Kirillov, A. A. Korotkevich, and S. G. Rubin, "Emergence of symmetries," Physics Letters B, vol. 718, no. 2, pp. 237-240, 2012.

[29] S. Weinberg, "The cosmological constant problem," Reviews of Modern Physics, vol. 61, no. 1, pp. 1-23, 1989.

[30] J. Martin, "Everything you always wanted to know about the cosmological constant problem (but were afraid to ask)," Comptes Rendus Physique, vol. 13, no. 6-7, pp. 566-665, 2012.

[31] Ya. B. Zeldovich, "Cosmological constant and elementary particles," JETP Letters, vol. 6, article 316, 1967.

[32] S. Nasri, P. J. Silva, G. D. Starkman, and M. Trodden, "Radion stabilization in compact hyperbolic extra dimensions," Physical Review D: Particles, Fields, Gravitation and Cosmology, vol. 66, no. 4, Article ID 045029, 2002.

[33] S. M. Carroll, J. Geddes, M. B. Hoffman, and R. M. Wald, "Classical stabilization of homogeneous extra dimensions," Physical Review D: Particles, Fields, Gravitation and Cosmology, vol. 66, no. 2, article 024036, 2002.
[34] B. Greene and J. Levin, "Dark energy and stabilization of extra dimensions," Journal of High Energy Physics, vol. 2007, no. 11, article no. 096, 2007.

[35] B. P. Abbott, R. Abbott, and T. D. Abbott, "Observation of gravitational waves from a binary black hole merger," Physical Review Letters, vol. 116, no. 6, article 061102, 2016.

[36] H. Yu, B.-M. Gu, F. P. Huang, Y.-Q. Wang, X.-H. Meng, and Y.-X. Liu, "Probing extra dimension through gravitational wave observations of compact binaries and their electromagnetic counterparts," Journal of Cosmology and Astroparticle Physics, vol. 2017, no. 2, article no. 039, 2017.

[37] D. Bettoni, J. M. Ezquiaga, K. Hinterbichler, and $M$. Zumalacárregui, "Speed of gravitational waves and the fate of scalar-tensor gravity," Physical Review D: Particles, Fields, Gravitation and Cosmology, vol. 95, no. 8, Article ID 084029, 2017.

[38] J. García-Bellido, S. Nesseris, and M. Trashorras, “Gravitational wave source counts at high redshift and in models with extra dimensions," Journal of Cosmology and Astroparticle Physics, vol. 2016, no. 7, article no. 021, 2016.

[39] E. Calabrese, N. Battaglia, and D. N. Spergel, “Testing gravity with gravitational wave source counts," Classical and Quantum Gravity, vol. 33, no. 16, Article ID 165004, 2016.

[40] B. Emanuele, K. Yagi, and Y. Nicolas, "Extreme Gravity Tests with Gravitational Waves from Compact Binary Coalescences: (I) Inspiral-Merger," General Relativity and Quantum Cosmology, https://arxiv.org/abs/1801.03208vl.

[41] K. A. Bronnikov, R. V. Konoplich, and S. G. Rubin, "The diversity of universes created by pure gravity," Classical and Quantum Gravity, vol. 24, no. 5, pp. 1261-1277, 2007. 

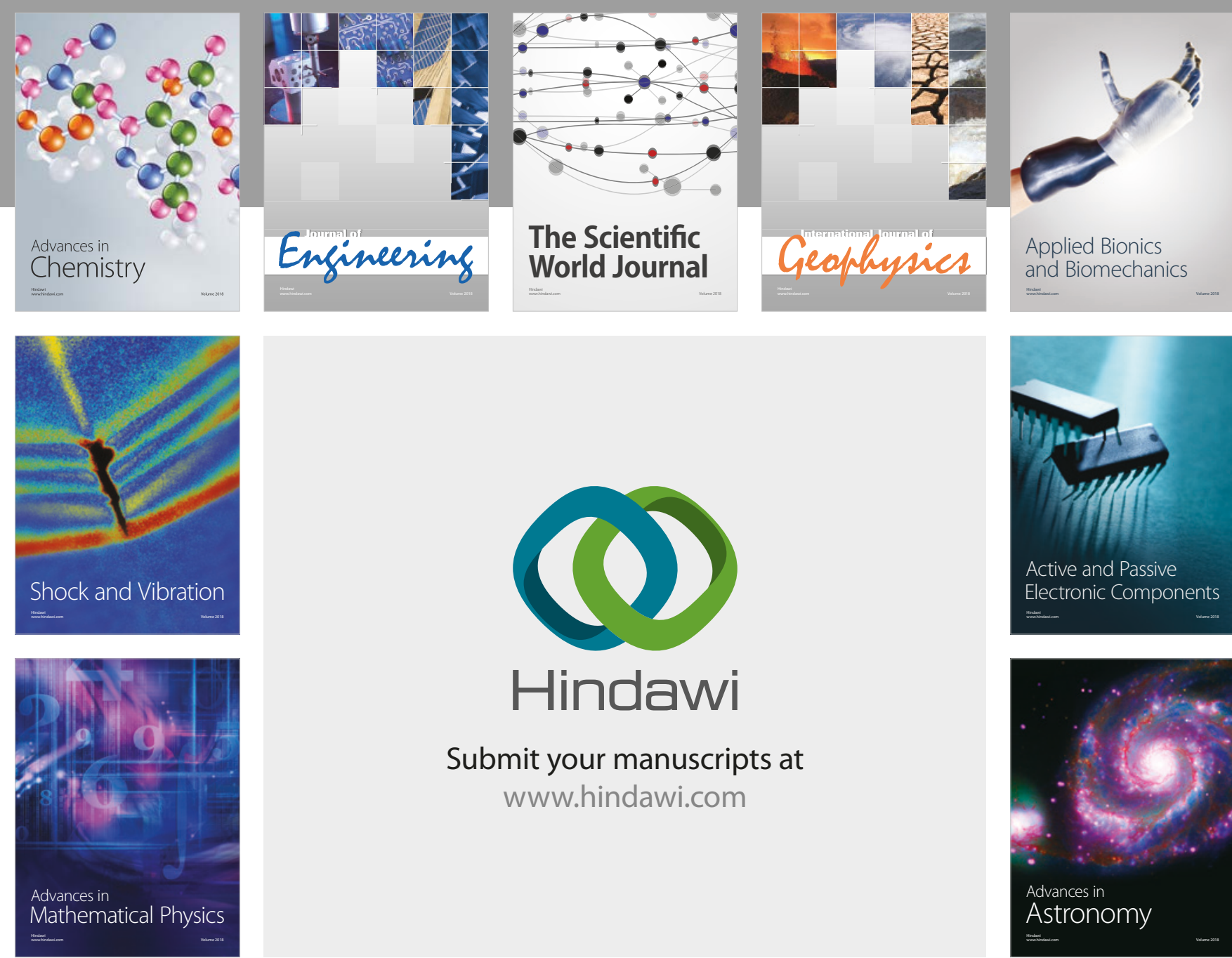

Submit your manuscripts at

www.hindawi.com

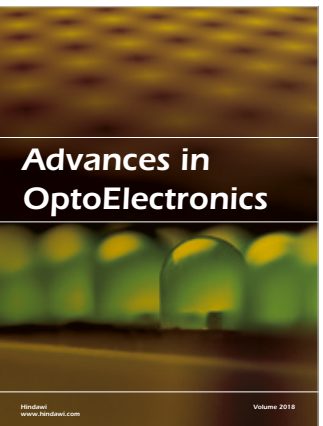

\section{Rotcting Machinery}
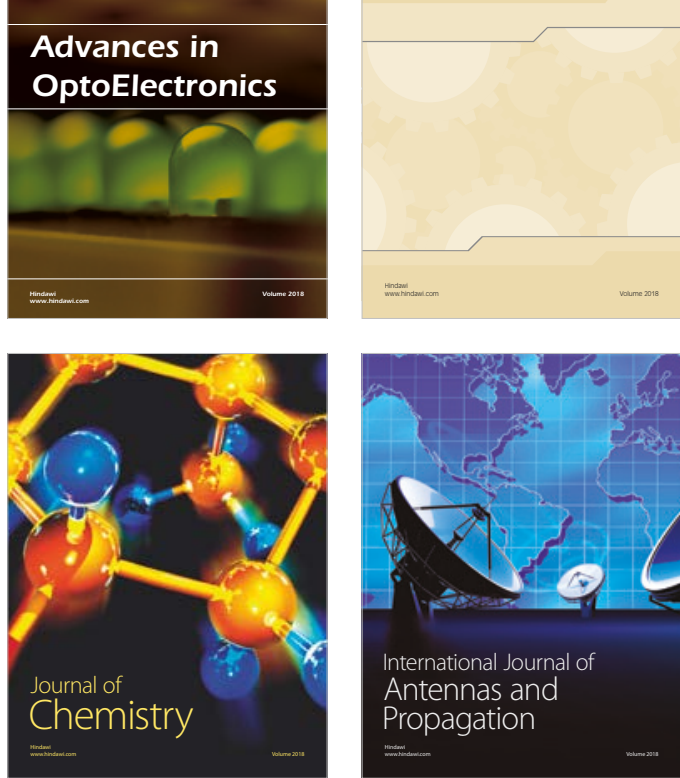

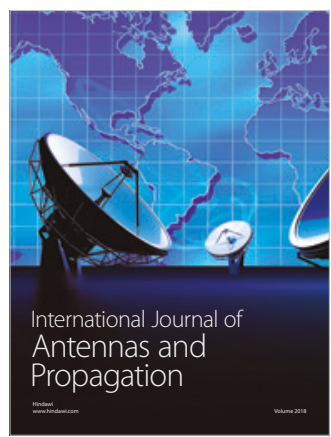

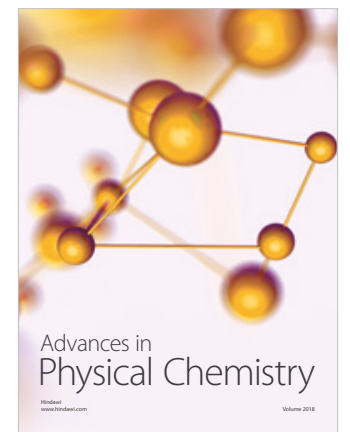

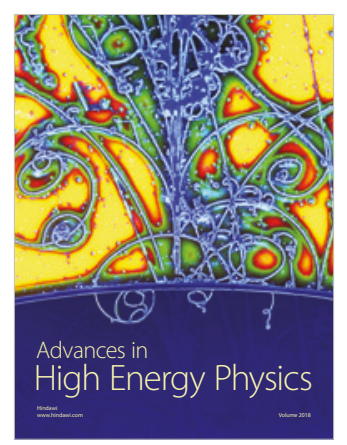

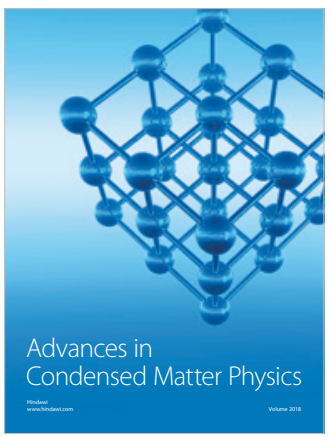

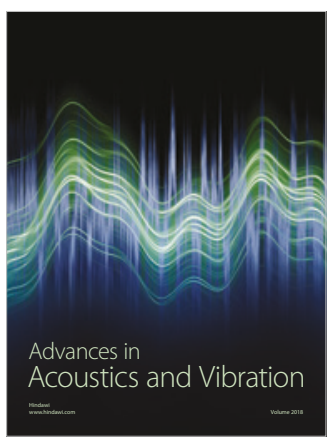

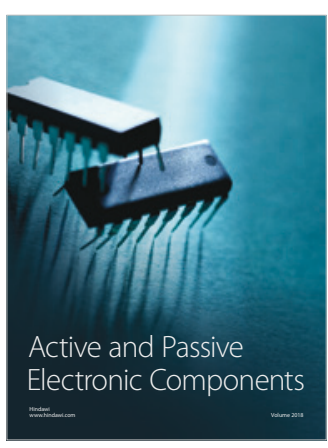
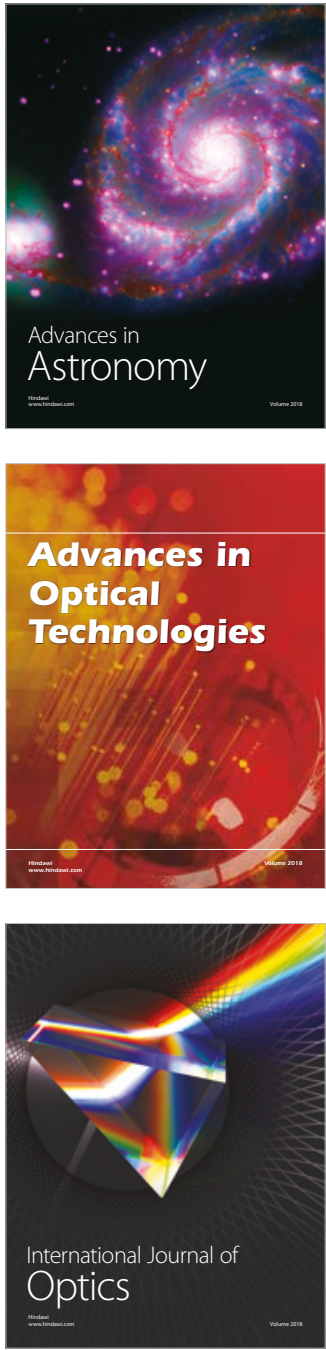